\title{
Article \\ A Case Series of Diarrheal Diseases Associated with Yersinia frederiksenii
}

\author{
Eugene Y. H. Yeung
}

check for

updates

Citation: Yeung, E.Y.H. A Case Series of Diarrheal Diseases Associated with Yersinia frederiksenii. Infect. Dis. Rep. 2021, 13, 552-557. https://doi.org/10.3390/idr13020051

Academic Editor: Nicola Petrosillo

Received: 9 May 2021

Accepted: 11 June 2021

Published: 13 June 2021

Publisher's Note: MDPI stays neutral with regard to jurisdictional claims in published maps and institutional affiliations.

Copyright: (C) 2021 by the author. Licensee MDPI, Basel, Switzerland. This article is an open access article distributed under the terms and conditions of the Creative Commons Attribution (CC BY) license (https:// creativecommons.org/licenses/by/ $4.0 /)$.
Department of Medical Microbiology, The Ottawa Hospital General Campus, The University of Ottawa, Ottawa, ON K1H 8L6, Canada; eugeney@doctors.org.uk

\begin{abstract}
To date, Yersinia pestis, Yersinia enterocolitica, and Yersinia pseudotuberculosis are the three Yersinia species generally agreed to be pathogenic in humans. However, there are a limited number of studies that suggest some of the "non-pathogenic" Yersinia species may also cause infections. For instance, Yersinia frederiksenii used to be known as an atypical Y. enterocolitica strain until rhamnose biochemical testing was found to distinguish between these two species in the 1980s. From our regional microbiology laboratory records of 18 hospitals in Eastern Ontario, Canada from 1 May 2018 to 1 May 2021, we identified two patients with Y. frederiksenii isolates in their stool cultures, along with their clinical presentation and antimicrobial management. Both patients presented with diarrhea, abdominal pain, and vomiting for 5 days before presentation to hospital. One patient received a 10-day course of sulfamethoxazole-trimethoprim; his $Y$. frederiksenii isolate was shown to be susceptible to amoxicillin-clavulanate, ceftriaxone, ciprofloxacin, and sulfamethoxazoletrimethoprim, but resistant to ampicillin. The other patient was sent home from the emergency department and did not require antimicrobials and additional medical attention. This case series illustrated that diarrheal disease could be associated with $Y$. frederiksenii; the need for antimicrobial treatment should be determined on a case-by-case basis.
\end{abstract}

Keywords: Yersinia frederiksenii; Yersinia enterocolitica; yersiniosis; diarrhea; microbial sensitivity tests; stool culture; sulfamethoxazole-trimethoprim; gastroenteritis

\section{Introduction}

Yersiniosis is a foodborne infectious disease that causes fever, abdominal pain, and often bloody diarrhea, which generally develop 4 to 7 days after exposure and last 1 to 3 weeks [1,2]. Yersiniosis is usually acquired by the ingestion and handling of contaminated food, especially raw or undercooked pork, contaminated milk, and untreated water [2,3]. Yersinia enterocolitica, and less often Yersinia pseudotuberculosis, are gram-negative bacterial species that cause human intestinal yersiniosis [2]. Pigs are the major animal reservoir of these species, although rabbits, sheep, cattle, horses, dogs, and cats can also carry strains that infect humans [2]. For hospital inpatients, the Centers for Disease Control and Prevention (CDC) recommends contact precautions for diapered or incontinent persons for the duration of illness or to control outbreaks [4]. However, yersiniosis is an uncommon disease in Canada, accounting for 288 cases (rate of 2 per 100,000), 15 hospitalizations, and no deaths in Ontario, Canada in 2019 [1]. Yersiniosis is generally a self-limiting disease that requires mainly supportive therapy; antibiotics are reserved for complicated infections, such as sepsis [2,3]. In a study of 67 patients with an $Y$. enterocolitica infection, antimicrobial treatment was not associated with a decreased duration of illness (18 and 21 days with and without antimicrobials, respectively) [5]. Therefore, antimicrobial susceptibility testing for Yersinia species is not always warranted.

To date, Yersinia pestis, Y. enterocolitica, and Y. pseudotuberculosis are the three Yersinia species generally agreed to be pathogenic in humans. They share a virulence plasmid (called pCD1 in Y. pestis and pYV in Y. enterocolitica and Y. pseudotuberculosis) and a chromosomal high-pathogenicity island (HPI), whereas the rest of the species in the Yersinia 
genus lack these virulence plasmid and chromosomal proteins [6]. Although Y. pestis does not generally cause gastrointestinal symptoms, it is the etiologic pathogen that causes life-threatening plague, which is usually transmitted to humans by the bite of an infected rat flea vector $[3,6]$. Y. pestis infection can also be transmitted through contact with a fluid or tissue from an infected animal and inhaling droplets from patients with pulmonary infection $[3,4]$. The CDC recommends droplet precaution for the source patient with pneumonic plague until $48 \mathrm{~h}$ after effective antibiotic therapy [4].

There were a limited number of published studies that suggested the "non-pathogenic" Yersinia species might also cause symptomatic infections in humans. For instance, in a study of 38 patients with non-pathogenic Yersinia species isolates (12 Yersinia intermedia, 7 Yersinia frederiksenii, 3 Yersinia kristensenii, and 16 unclassified Yersinia isolates), 10 patients had diarrhea alone [7]. The diarrheal symptoms persisted for less than a week in $21 \%$ and more than a month in $54 \%$ of the cases. Other contributory factors to their symptoms included prior use of antimicrobials and concurrent use of corticosteroids and proton pump inhibitors.

In our microbiology laboratory in Eastern Ontario Regional Laboratory Association (EORLA), we retrospectively audited stool cultures in the eighteen affiliated hospitals. After reviewing all of the patients with Yersinia isolates in their stool cultures from 1 May 2018 to 1 May 2021 (3-year inclusive), we identified eleven patients-two with Y. frederiksenii and nine with $Y$. enterocolitica isolates. The current quality improvement project detailed the clinical presentations of patients with $Y$. frederiksenii isolates in their stool cultures, which are generally known as non-pathogens [6]. The current project aimed to determine how antimicrobial susceptibility testing and treatment impacted patients' clinical management.

\section{Materials and Methods}

The current quality improvement project followed the guidelines and standards given by the Ottawa Health Science Network Research Ethics Board. Eighteen hospitals in Eastern Ontario (the Almonte General Hospital, Arnprior Regional Health, Carleton Place and District Memorial Hospital, Children's Hospital of Eastern Ontario, Cornwall Community Hospital, Deep River and District Hospital, Glengarry Memorial Hospital, Hawkesbury District General Hospital, Kemptville District Hospital, Montfort Hospital, Pembroke Regional Hospital, Queensway Carleton Hospital, Renfrew Victoria Hospital, St. Francis Memorial Hospital, Ottawa General Hospital, Ottawa Civic Hospital, Ottawa Riverside Hospital, and Winchester District Memorial Hospital) submitted patients' stool samples to the EORLA regional microbiology laboratory for testing. The laboratory used the Cerner Millennium software (Version 2013.04.1.34; Kansas City, MO, USA) to store the patients' laboratory data. This software generated reports that included all of the patients with Yersinia species isolated in their stool cultures in a three-year period (1 May 2018 to 1 May 2021). To reduce the risk of false positive and negative results, the microbiology laboratory would only perform cultures on the patients' stool specimens if they were transported to with Cary Blair Transport Medium, which had been internally validated for use.

The stool specimens were cultured on MacConkey agar plates at $35{ }^{\circ} \mathrm{C}$ for $24 \mathrm{~h}$ in an oxygen incubator, where colonies of non-lactose fermenting microorganisms (such as Salmonella, Shigella, and Yersinia species) would be suggestive of stool pathogens and thereby undergo further testing. To improve the yield of Yersinia species, the MacConkey plates were left at room temperature for an additional $24 \mathrm{~h}$, where pinpoint colonies would be suggestive of stool pathogens and thereby undergo further testing. To improve the yield of Salmonella and Shigella species, the stool specimens were cultured on Hektoen selective agar plates at $35^{\circ} \mathrm{C}$ for $24 \mathrm{~h}$ in an oxygen incubator. To improve the yield of Escherichia coli O157, the stool specimens were also cultured on sorbitol MacConkey selective agar plates $35{ }^{\circ} \mathrm{C}$ for $24 \mathrm{~h}$ in an oxygen incubator. To improve the yield of Campylobacter species, the stool specimens were also cultured on Campylobacter selective agar plates at $42{ }^{\circ} \mathrm{C}$ for $48 \mathrm{~h}$ in sealed jars with CamyGen sachets. The microorganisms isolated from the above agar plates were further identified using Bruker matrix-assisted laser desorption ionization-time of 
flight mass spectrometry (MALDI-TOF MS). Each microorganism was identified to species level when the MALDI-TOF score was $\geq 2.0$. The antimicrobial susceptibility results were based on Clinical and Laboratory Standards Institute (CLSI) Kirby-Bauer inhibition zone breakpoints. However, antimicrobial susceptibility testing was not routinely performed on Yersinia isolates in stools unless specifically requested by the clinicians, as there is a lack of evidence to support the benefits of antimicrobial therapy [5].

Each patient's electronic health record was retrospectively reviewed using the software Epic Hyperspace (Version November 2018, Verona, MI, USA). Their demographics, clinical history, and therapeutic records were recorded on a separate spreadsheet.

\section{Results}

Patient A was a 66-year-old male with a history of asthma, chronic obstructive pulmonary disease, depression, gastroesophageal reflux disease, myocardial infarction, pulmonary fibrosis, and substance use disorder, who resided in a homeless shelter. He had Clostridioides difficile diarrhea diagnosed using a C. difficile toxin B polymerase chain reaction (PCR) assay about a month prior to his current hospital admission and was treated with 21 days of oral vancomycin and 10 days of intravenous metronidazole. He presented to hospital again with vomiting, loose stool, and abdominal pain for 5 days when he was not on antimicrobials. He was afebrile and hemodynamically stable. His bloodwork results on arrival were as follows: white blood cell count $10.3 \times 10^{9} / \mathrm{L}$; platelet $362 \times 10^{9} / \mathrm{L}$; sodium $131 \mathrm{mmol} / \mathrm{L}$; creatinine $72 \mathrm{umol} / \mathrm{L}$; potassium $3.6 \mathrm{mmol} / \mathrm{L}$; and lactate $2.4 \mathrm{mmol} / \mathrm{L}$. Computed tomography images of his abdomen and pelvis revealed dilated small and large bowels and increased intraluminal fluid, suggestive of enterocolitis. His stool sample was processed in the microbiology laboratory, which reported negative C. difficile PCR but growth of $Y$. frederiksenii on the culture plates. No other stool pathogens were identified. At that time, the patient had been having ongoing diarrhea in hospital at least three times a day for four days. The infectious diseases team was consulted and requested antimicrobial susceptibility testing. The patient was placed on contact precaution because of his acute gastroenteritis symptoms. In view of the patient's ongoing symptoms, the infectious diseases team opted to start the patient on one sulfamethoxazole-trimethoprim double strength tablet $(800 \mathrm{mg} / 160 \mathrm{mg}$ per tablet) twice a day for 10 days. Antimicrobial susceptibility testing was performed on his $Y$. frederiksenii isolate with Kirby-Bauer discs (Table 1). For the purpose of antimicrobial stewardship [8], our laboratory cascade reported the isolate's susceptibility to amoxicillin-clavulanate, ceftriaxone, ciprofloxacin, and sulfamethoxazole-trimethoprim, and resistance to ampicillin only. No change was made to Patient A's antimicrobial therapy as his $Y$. frederiksenii isolate was susceptible to sulfamethoxazole-trimethoprim. On day five of the sulfamethoxazole-trimethoprim therapy, the patient was discharged home in a stable condition. His blood cultures, Helicobacter pylori IgG, stool ova, and parasite testing results were found to be negative later. The patient re-visited the emergency department 4 days after hospital discharge because of mood and housing issues. During the visit, the patient denied having diarrhea, suggesting the sulfamethoxazole-trimethoprim therapy was effective in curing his diarrheal illness.

Patient B was a 35-year-old female with a history of anxiety and gastroesophageal reflux disease (H. pylori IgG non-reactive) who presented with diarrhea, abdominal pain, nausea, and vomiting for 5 days. She was afebrile and hemodynamically stable. No bloodwork and radiological imaging were ordered. She was given one dose of $50 \mathrm{mg}$ dimenhydrinate and sent home from the emergency department. Her stool ova and parasite testing were negative, but a culture showed $Y$. frederiksenii isolates. No other stool pathogens were identified. The emergency department staff gave the patient a phone call 4 days after her visit and disclosed the microbiological diagnosis. However, she required no antimicrobials or further medical attention. Antimicrobial susceptibility testing was not performed on her $Y$. frederiksenii isolates. 
Table 1. Antimicrobial susceptibility of Patient A's Y. frederiksenii isolate in stool culture (with Kirby-Bauer zone of inhibition diameter in millimeters) *.

\begin{tabular}{ccc}
\hline Gentamicin & Susceptible & $27 \mathrm{~mm}$ \\
Tobramycin & Susceptible & $28 \mathrm{~mm}$ \\
Amikacin & Susceptible & $25 \mathrm{~mm}$ \\
Amoxicillin-clavulanate & Susceptible & $18 \mathrm{~mm}$ \\
Ampicillin & Resistant & $7 \mathrm{~mm}$ \\
Cefazolin & Resistant & $14 \mathrm{~mm}$ \\
Ceftriaxone & Susceptible & $32 \mathrm{~mm}$ \\
Ciprofloxacin & Susceptible & $37 \mathrm{~mm}$ \\
Ertapenem & Susceptible & $38 \mathrm{~mm}$ \\
Meropenem & Susceptible & $35 \mathrm{~mm}$ \\
Nitrofurantoin & Susceptible & $21 \mathrm{~mm}$ \\
Piperacillin-tazobactam & Susceptible & $30 \mathrm{~mm}$ \\
Sulfamethoxazole- & Susceptible & $31 \mathrm{~mm}$ \\
trimethoprim & Susceptible & $29 \mathrm{~mm}$ \\
Ceftazidime & Intermediate & $20 \mathrm{~mm}$ \\
Cefpodoxime & &
\end{tabular}

\section{Discussion}

Y. frederiksenii used to be known as an atypical Y. enterocolitica strain until rhamnose biochemical testing was found to distinguish between these two species in the 1980s [9]. Y. frederiksenii could be differentiated from Y. pseudotuberculosis by showing positive reactions for ornithine decarboxylase, indole, sucrose, sorbose, sorbitol, inositol, and VogesProskauer [9]. As evidenced in this case series, Y. frederiksenii could also cause diarrheal diseases such as $Y$. enterocolitica and $Y$. pseudotuberculosis, the two etiologic agents for yersiniosis. Y. frederiksenii could be contributory to the 5 days of abdominal pain and vomiting these two patients experienced. As evidenced in Patient A, untreated Y. frederiksenii might lead to complications such as enterocolitis and a high lactate level.

Nevertheless, the presence of $Y$. frederiksenii in stools does not always warrant the use of antimicrobials. Patient $B$ required no antimicrobials or further medical attention, despite being symptomatic at initial presentation with $Y$. frederiksenii found in the stool culture. This finding was consistent with a study of 38 patients with non-pathogenic Yersinia species isolates (12 Y. intermedia, 7 Y. frederiksenii, 3 Y. kristensenii, and 16 unclassified Yersinia isolates), where only five patients required antimicrobial treatment; two patients received ciprofloxacin that resulted in symptoms for more than 1 month; one patient received erythromycin that resulted in symptoms for less than a week; and two patients received metronidazole and had no follow-ups. [7]. However, it was unclear which Yersinia species these five patients contracted.

The fact that Yersinia diarrheal diseases may self-resolve justifies why our microbiology laboratory does not routinely perform antimicrobial susceptibility testing on Yersinia isolates in stool, unless requested by clinicians. If antimicrobial susceptibility tests were performed for all patients, their clinicians might be misled to prescribe antimicrobials even if they are not needed. Patient A's Y. frederiksenii isolates showed susceptibility to amoxicillin-clavulanate, ceftriaxone, ciprofloxacin, and sulfamethoxazole-trimethoprim, but resistance to ampicillin. The findings were consistent with a study of 38 Y. frederiksenii strains collected in France, Germany, Sweden, Switzerland, and Norway, where 36 strains were susceptible to amoxicillin-clavulanate, all of the strains were susceptible to ceftriaxone, ciprofloxacin, and sulfamethoxazole-trimethoprim, and all of the strains were resistant to ampicillin or amoxicillin (as per the CLSI minimum inhibitory concentration breakpoints) [10]. However, the need for antimicrobial treatment should be determined on a case-by-case basis.

Patient A was put on contact precaution due to his gastroenteritis symptoms. There is currently a lack of evidence to suggest that $Y$. frederiksenii could be transmitted through 
direct person-to-person contact. An infection control study was conducted on a 24-year-old female hospital resident physician, who was diagnosed to have a gastrointestinal infection with $Y$. frederiksenii in 1993 [11]. Her nine co-residents were screened for fecal carriage of $Y$. frederiksenii-three of them were tested positive but none of them had symptoms. Despite carrying out surveillance studies on the symptomatic resident physician's unpasteurized milk and hospital holding tanks, the infection control team failed to identify the source of her $Y$. frederiksenii infection.

The current study added to the debate as to whether the "non-pathogenic" Yersinia species causes symptomatic infections in humans. Despite our best efforts, our regional laboratory captured only two cases of "non-pathogenic" Yersinia species isolated from stool cultures in a 36-month period. The rare incidence rate of Yersiniosis ( 2 per 100,000 in Ontario [1]) led to the small sample size, a major limitation of the current study. Moreover, our laboratory would only process cultures on the stool samples collected in Cary Blair Transport Medium. It is possible that there were more patients with yersiniosis whose stool samples were rejected in the laboratory due to the incorrect containers used. The current case patients did not undergo viral gastroenteritis testing; a positive result would suggest co-infection or an alternative diagnosis. Nevertheless, viral gastroenteritis tends to be a self-limiting disease in immunocompetent adults that do not always warrant laboratory investigations in clinical practice [12]. Unlike previous studies on non-pathogenic Yersinia species $[7,10]$, the current quality improvement project was specific to $Y$. frederiksenii. Despite the small sample size, the current case series demonstrated that $Y$. frederiksenii isolates in stool did not always require antimicrobial treatment. The antimicrobial susceptibility profile of $Y$. frederiksenii in our laboratory in Eastern Ontario, Canada, was consistent with the published data in Europe [10].

Future quality improvement projects should try to capture more cases of Yersinia isolates in stool. This could be performed by a continual auditing of the microbiology laboratory stool culture results, along with patients' clinical histories and therapeutic records. The laboratory may consider the use of selective agar plates specific for Yersinia species to further improve the positivity rate [13-15]. Better education and communication with the clinicians may improve the use of correct containers to transport stool for culturing.

\section{Conclusions}

The results were inconclusive on whether the $Y$. frederiksenii isolates in stool were pathogenic isolates that required antimicrobial treatment and isolation precaution. If antimicrobials are needed, $Y$. frederiksenii isolates have been shown to be susceptible to amoxicillin-clavulanate, ceftriaxone, ciprofloxacin, and sulfamethoxazole-trimethoprim, but resistant to ampicillin. More data is needed to help clinicians to determine the best management of $Y$. frederiksenii isolates in stools.

Funding: This quality improvement project received no external funding.

Institutional Review Board Statement: The data collected in the current study were from a quality improvement project that investigated the need for antimicrobial susceptibility testing (approved on 3 December 2019). The project was deemed to be a quality improvement project and followed the guidelines and standards given by the Ottawa Health Science Network Research Ethics Board.

Informed Consent Statement: Patient consent was waived because the data collected were from a retrospective quality improvement projects but not research study. Patient-identifiable information was anonymized in the current manuscript.

Data Availability Statement: The raw data presented in this study are available on request from the corresponding author. The data are not publicly available due to patient confidentiality.

Conflicts of Interest: The author has been paid for working as a medical doctor, but not for writing the manuscript. 


\section{References}

1. Public Health Ontario. Yersiniosis. Available online: https://www.publichealthontario.ca/en/diseases-and-conditions/ infectious-diseases/enteric-foodborne-diseases/yersiniosis (accessed on 21 April 2021).

2. Centers for Disease Control and Prevention (CDC). Yersinia Enterocolitica (Yersiniosis) Questions and Answers. Available online: https:/ / www.cdc.gov/yersinia/faq.html (accessed on 21 April 2021).

3. Bush, L.M.; Vazquez-Pertejo, M.T. Plague and Other Yersinia Infections. Available online: https://www.merckmanuals.com/en$\mathrm{ca} /$ professional/infectious-diseases/gram-negative-bacilli/plague-and-other-yersinia-infections (accessed on 21 April 2021).

4. Centers for Disease Control and Prevention (CDC). Type and Duration of Precautions Recommended for Selected Infections and Conditions. Available online: https://www.cdc.gov/infectioncontrol/guidelines/isolation/appendix/type-durationprecautions.html (accessed on 21 April 2021).

5. Ostroff, S.M.; Kapperud, G.; Lassen, J.; Aasen, S.; Tauxe, R.V. Clinical features of sporadic Yersinia enterocolitica infections in Norway. J. Infect. Dis. 1992, 166, 812-817. [CrossRef]

6. Petersen, J.M.; Gladney, L.M.; Schriefer, M.E. Yersinia. In Manual of Clinical Microbiology, 11th ed.; Jorgensen, J.H., Pfaller, M.A., Carroll, K.C., Funke, G., Landry, M.L., Richter, S.S., Warnock, D.W., Eds.; American Society of Microbiology: Washington, DC, USA, 2015; pp. 738-748.

7. Loftus, C.G.; Harewood, G.C.; Cockerill, F.R., 3rd; Murray, J.A. Clinical features of patients with novel Yersinia species. Dig. Dis. Sci. 2002, 47, 2805-2810. [CrossRef] [PubMed]

8. Liao, S.; Rhodes, J.; Jandarov, R.; DeVore, Z.; Sopirala, M.M. Out of sight—out of mind: Impact of cascade reporting on antimicrobial usage. Open Forum. Infect. Dis. 2020, 7, ofaa002. [CrossRef] [PubMed]

9. Ursing, J.; Don Brennert, J.; Bercovier, H.; Richard Fanning, G.; Steigerwalt, A.G.; Brault, J.; Mollaret, H.H. Yersinia frederiksenii: A new species of enterobacteriaceae composed of rhamnose-positive strains (formerly called atypical Yersinia enterocolitica or Yersinia enterocolitica-like). Curr. Microbiol. 1980, 4, 213-217. [CrossRef]

10. Stock, I.; Wiedemann, B. Natural antimicrobial susceptibilities and biochemical profiles of Yersinia enterocolitica-like strains: Y. frederiksenii, Y. intermedia, Y. kristensenii and Y. rohdei. FEMS Immunol. Med. Microbiol. 2003, 38, 139-152. [CrossRef]

11. Cafferkey, M.T.; Sloane, A.; McCrae, S.; O'Morain, C.A. Yersinia frederiksenii infection and colonization in hospital staff. J. Hosp. Infect. 1993, 24, 109-115. [CrossRef]

12. Barr, W.; Smith, A. Acute diarrhea in adults. Am. Fam. Physician 2014, 89, 180-189. [PubMed]

13. Fukushima, H. New selective agar medium for isolation of virulent Yersinia enterocolitica. J. Clin. Microbiol. 1987, 25, 1068-1073. [CrossRef] [PubMed]

14. Schiemann, D.A. Synthesis of a selective agar medium for Yersinia enterocolitica. Can. J. Microbiol. 1979, 25, 1298-1304. [CrossRef] [PubMed]

15. Weagant, S.D. A new chromogenic agar medium for detection of potentially virulent Yersinia enterocolitica. J. Microbiol. Methods 2008, 72, 185-190. [CrossRef] [PubMed] 